Check for updates

Cite this: Phys. Chem. Chem. Phys., 2020, 22, 8450

Received 22nd January 2020, Accepted 24th March 2020

DOI: 10.1039/d0cp00360c

rsc.li/pccp

\section{Interfacial structuring of non-halogenated imidazolium ionic liquids at charged surfaces: effect of alkyl chain length $\dagger$}

\author{
Seiya Watanabe, (D) $\ddagger^{a}$ Georgia A. Pilkington, (D) ${ }^{a}$ Anna Oleshkevych, ${ }^{a}$ \\ Patricia Pedraz, ${ }^{a}$ Milad Radiom, (D) ${ }^{\text {a }}$ Rebecca Welbourn, ${ }^{b}$ Sergei Glavatskih (D) ${ }^{c d}$ and \\ Mark W. Rutland (D) *aef
}

\begin{abstract}
Control of the interfacial structures of ionic liquids (ILs) at charged interfaces is important to many of their applications, including in energy storage solutions, sensors and advanced lubrication technologies utilising electric fields. In the case of the latter, there is an increasing demand for the study of nonhalogenated ILs, as many fluorinated anions have been found to produce corrosive and toxic halides under tribological conditions. Here, the interfacial structuring of a series of four imidazolium ILS $\left(\left[C_{n} C_{1} \mid m\right]\right)$ of varying alkyl chain lengths $(n=5,6,7,10)$, with a non-halogenated borate-based anion ([BOB]), have been studied at charged interfaces using sum frequency generation (SFG) spectroscopy and neutron reflectivity (NR). For all alkyl chain lengths, the SFG spectra show that the cation imidazolium ring responds to the surface charge by modifying its orientation with respect to the surface normal. In addition, the combination of SFG spectra with electrochemical NR measurements reveals that the longest alkyl chain length $(n=10)$ forms a bilayer structure at all charged interfaces, independent of the ring orientation. These results demonstrate the tunability of IL interfacial layers through the use of surface charge, as well as effect of the cation alkyl chain length, and provide valuable insight into the charge compensation mechanisms of ILs.
\end{abstract}

\section{Introduction}

Ionic liquids (ILs) have many properties, such as low vapour pressures and high thermal stability, which has led them to be extensively studied for a wide range of applications, including as lubricants or lubricant additives. ${ }^{1}$ Moreover, the interfacial structures of ILs can be modified through control of the surface charge, providing the potential advantage of tribotronics,

\footnotetext{
${ }^{a}$ Division of Surface and Corrosion Science, School of Engineering Sciences in Chemistry, Biotechnology and Health, KTH Royal Institute of Technology, SE-100 44 Stockholm, Sweden. E-mail: mark@kth.se

${ }^{b}$ ISIS Neutron \& Muon Source, Rutherford Appleton Laboratory, STFC, Chilton, Didcot, OXON OX11 OQX, UK

'System and Component Design, Department of Machine Design, KTH Royal Institute of Technology, SE-100 44 Stockholm, Sweden

${ }^{d}$ Department of Electrical Energy, Metals, Mechanical Constructions and Systems, Ghent University, B-9052 Ghent, Belgium

${ }^{e}$ Surfaces, Processes and Formulation, RISE Research Institutes of Sweden, SE-100 44 Stockholm, Sweden

${ }^{f}$ Laboratoire de Tribologie Dynamique des Systèmes,

36 Avenue Guy de Collongue Ecole Centrale de Lyon, 69134 Écully, France

$\dagger$ Electronic supplementary information (ESI) available. See DOI: 10.1039/d0cp00360c

\# Current address: Tokyo University of Science, Department of Mechanical

Engineering, 6-3-1 Niijuku, Katsushika-ku, Tokyo 125-8585, Japan.
}

whereby the active control of friction is realised using an electric field, ${ }^{2}$ as first demonstrated by Sweeney et al. ${ }^{3}$ However, the majority of previous studies have focused on halogenated anions, which in recent years have come under scrutiny due to environmental and corrosion concerns. For example, halogenated anions can lead to severe corrosive wear under tribological conditions, ${ }^{4}$ i.e. high pressures and temperatures, leading to shortened lifetimes of sliding components. Hence, halogen-free ILs are desirable for lubricant applications. Recently, ILs with boron-based anions, which exhibit excellent frictional properties, e.g. lower friction coefficient and wear rates than a commercial reference oil, have been successfully synthesised. ${ }^{5,6}$ However, their friction reduction mechanisms are still to be fully understood.

It is well known that the presence of interfacial molecular structures can greatly contribute to friction reduction by preventing direct contact of asperities between two sliding surfaces in boundary lubrication. Therefore, characterisation of molecular ordering at the solid surface is crucial to the understanding of the friction reduction mechanism. Many typical cation species of ILs, including imidazolium, pyrrolidinium, ammonium and phosphonium ions, consist of a ring or cation centre decorated with alkyl chains. ${ }^{7}$ It has long been known that the length of the alkyl chains in lubricant boundary layers can play an important role 
in their friction reduction. In particular, longer alkyl chain cations are likely to form more densely packed layers due to van der Waals forces between the alkyl chains which, under tribological conditions, can prevent the breakdown of boundary film by friction forces. ${ }^{8,9}$

Previous tribotronic IL studies have also reported that differences in cation alkyl chain lengths strongly affect their frictional properties and electric response. ${ }^{10,11}$ In addition, when the length of the alkyl chain of the cation becomes significant, other effects can be driven. Previous measurements using the surface force apparatus (SFA) by Perkin et al. have shown a transition from a monolayer to bilayer structure in $\left[\mathrm{C}_{4} \mathrm{C}_{1} \mathrm{Im}\right]\left[\mathrm{NTf}_{2}\right]$ and $\left[\mathrm{C}_{6} \mathrm{C}_{1} \mathrm{Im}\right]\left[\mathrm{NTf}_{2}\right]$ imidazoliumbased fluorinated ILs, respectively, when they are confined between two negatively charged mica surfaces. ${ }^{12}$ Neutron reflectivity (NR) measurements by Griffin et al. have also shown bilayer structuring of another imidazolium-based halogenated IL with a longer alkyl chain cation $\left(\left[\mathrm{C}_{10} \mathrm{C}_{1} \mathrm{Im}\right]\left[\mathrm{NTf}_{2}\right]\right)$ on an unconfined mica surface. ${ }^{13}$ Such bilayer-like structures have been previously shown to have enhanced load carrying capacity, and thus promising lubricating properties. ${ }^{14-16}$ For example, Smith et al. performed SFA measurements on $\left[\mathrm{C}_{10} \mathrm{C}_{1}\right.$ Pyrr $]\left[\mathrm{NTf}_{2}\right]$ and $\left[\mathrm{C}_{4} \mathrm{C}_{1}\right.$ Pyrr $]\left[\mathrm{NTf}_{2}\right]$, and demonstrated that the friction coefficient associated with two bilayers of $\left[\mathrm{C}_{10} \mathrm{C}_{1} \mathrm{Pyrr}\right]\left[\mathrm{NTf}_{2}\right]$ resulted in extremely low friction in comparison to that measured for a shorter-chain, monolayer forming analogue $\left(\left[\mathrm{C}_{4} \mathrm{C}_{1} \mathrm{Pyrr}\right]\left[\mathrm{NTf}_{2}\right]\right){ }^{17}$ From these results, it was hypothesised that in the case of a bilayer-bilayer interaction, a slip plane between the cation methylimidazolium rings provides much lower friction than that between alkyl chains, analogous to that found for surfactants and phospholipids. ${ }^{18}$

Most previous studies of ILs with long alkyl chain cations have focused on those with halogenated anions, and halogenfree analogues are yet to be thoroughly investigated, though there are exceptions. ${ }^{19,20}$ In addition, SFA measurements, with their exquisite accuracy in determining zero separation, are typically limited to negatively charged mica as a substrate, whilst positively charged surfaces remain largely unexplored. ${ }^{21,22}$ In terms of tribotronics, it is crucial to understand the molecular behaviour of ILs at both positively and negatively charged surfaces. In a recent study, Baldelli et al. reported that the orientation of the imidazolium ring at the solid-liquid interface changes depending on the surface charge. ${ }^{23,24}$ In a related tribological study, the orientation tendency of the imidazolium ring has also been shown to have a strong correlation with the frictional properties of imidazolium-based ILs. ${ }^{25}$ Tribotronic colloidal probe AFM measurements have demonstrated imidazolium cations bearing longer alkyl chains to provide greater cohesiveness under applied load, particularly at negative potentials, due to the increased solvophobic interactions between the cation alkyl chains. ${ }^{26}$

It is clear that both the cation alkyl chain length and surface charge in imidazolium ILs will influence the interfacial structuring of the ILs at the solid-liquid interface, as well as determine their frictional and tribotronic properties. However, so far, orientation analysis of imidazolium-based ILs at the solid-liquid interface by sum-frequency generation (SFG) spectroscopy has been limited to those with shorter alkyl chains, such as $\left[\mathrm{C}_{4} \mathrm{C}_{1} \mathrm{Im}\right]$. Hence, whether such orientation behaviour would persist for longer chain analogues is yet to be clarified.
Here, SFG measurements have been performed on ILs at positively and negatively charged salt surfaces to mimic electrified surfaces. ${ }^{27,28}$ The ILs investigated are composed of a boron-based anion, which has shown promising friction-reducing properties, 5 and imidazolium cations decorated with different alkyl chain lengths $\left(\left[\mathrm{C}_{n} \mathrm{C}_{1} \mathrm{Im}\right][\mathrm{BOB}] ; n=5-7,10\right)$. Recent spectroscopic studies on the stretching modes of the imidazolium ring have revealed that the original vibrational modes of aromatic $\mathrm{C}-\mathrm{H}$ overlap with several Fermi resonance peaks. ${ }^{29,30}$ Hence, SFG measurement of the $\mathrm{C}-\mathrm{H}$ stretching modes of the $\mathrm{IL}$ imidazolium rings enables determination of the orientational tendency, rather than the specific orientation angle. To resolve this ambiguity, electrochemical (EC) NR measurements were also performed on the longest chain IL $(n=10)$ which allowed the surface potential to be systematically varied in situ and the resulting interfacial structures normal to the interface (z-direction) to be characterised. Such EC-NR measurements in ILs or IL solutions are relatively novel, and have only been performed in a limited number of studies. ${ }^{31-34}$ By obtaining the $z$-profile of the IL at comparable surface charges to those studied with SFG, and by exploiting the different neutron scattering properties of the ionic species, the anticipated orientation changes of the imidazolium ring with surface charge were verified and the extended interfacial structuring of the IL into the bulk also examined.

\section{Experimental}

\subsection{Materials}

The ILs 1-alkyl-3-methyl imidazolium bis(oxalato)borate, $\left[\mathrm{C}_{n} \mathrm{C}_{1} \mathrm{Im}\right]$ [BOB] $(n=5,6,7,10)$ were synthesised at Luleå University of Technology. ${ }^{35,36}$ The molecular structures of the ILs are shown in Fig. 1. All the ILs were dried for 68 hours under vacuum (12 mbar) at maximum temperature of $60{ }^{\circ} \mathrm{C} . \mathrm{BaF}_{2}(111)$ and $\mathrm{NaCl}(100)$ crystals were used as both window and substrate for the SFG measurements and purchased from United Crystals Inc. and Koch Crystal Finishing Inc., respectively.

$\mathrm{BaF}_{2}(111)$ and $\mathrm{NaCl}(100)$ mimic surface charging due to the relaxation of the surface atoms. The topmost surface of $\mathrm{BaF}_{2}(111)$ is barium-enriched, resulting in an overall dipolar structure with a local field reminiscent of a positive surface charge, whilst the topmost surface of $\mathrm{NaCl}(100)$, is chlorideenriched, giving rise to an opposite field. ${ }^{37,38}$ It can be anticipated that this effect may be influenced by the medium with which the surface interacts. Nonetheless, following previous related works of such surfaces in ILs, we assume that that the $\mathrm{BaF}_{2}(111)$ and

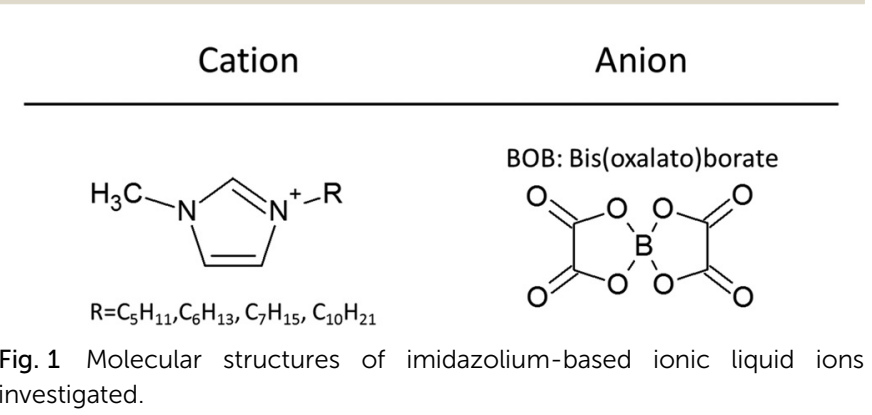

Fig. 1 Molecular structures of imidazolium-based ionic liquid ions investigated 
$\mathrm{NaCl}(100)$ interfaces preserve their positive and negative behaviour, respectively, in the manner demonstrated by Baldelli et al. ${ }^{27,28}$ The maximal surface charge densities of solid crystals can be estimated using lattice parameter of the crystals. ${ }^{27}$ These calculated, limiting, surface charge densities are $42 \mu \mathrm{C} \mathrm{cm}^{-2}$ for $\mathrm{BaF}_{2}(111)$ and $-50 \mu \mathrm{C} \mathrm{cm}^{-2}$ for $\mathrm{NaCl}(100)$.

\subsection{Sum-frequency generation (SFG) spectroscopy}

The theory of SFG spectroscopy has been reported elsewhere, ${ }^{39-41}$ and thus details are omitted here. A homemade liquid SFG cell ${ }^{42}$ was used for the measurement of interfacial structure of a series of ILs at the solid-liquid interface as shown in Fig. 2.

The cell was rinsed with isopropanol, acetone and ethanol, and dried with filtered $\mathrm{N}_{2}$. It was prepared and sealed in a glove box filled with $\mathrm{N}_{2}(\mathrm{RH}<10 \%)$ to minimise water absorption, which for other IL systems has been shown to have a strong influence on the interfacial structuring at charged interfaces. ${ }^{43-46}$ The SFG spectroscopy system in the present study used a Nd:YAG laser generating a mode-locked $1064 \mathrm{~nm}$ fundamental pulse beam for $30 \mathrm{ps}$ at a rate of $20 \mathrm{~Hz}$. Fixed visible $(532 \mathrm{~nm})$ and tuneable infrared $(2.5-10 \mu \mathrm{m})$ beams were produced by second harmonic generation (SHG) and optical parametric generation (OPG). The visible (VIS) and infrared (IR) beams overlapped at the crystal/IL interface with incident angles of $55^{\circ}$ and $63^{\circ}$ from the surface normal, respectively. The representative input energies of the visible and infrared beams were set to $\sim 150$ and $\sim 250 \mu \mathrm{J}$ per pulse, respectively. SSP and PPP polarisation combinations (of SFG, visible, infrared light) were used. $P$ represents light with the electric field polarised parallel to the incident plane and $S$ represents light polarised perpendicular to the plane.

\subsection{Neutron reflectivity (NR)}

2.3.1 Neutron reflectivity measurements and data analysis. The NR measurements presented here were performed on the INTER reflectometer at the ISIS Facility, Rutherford Appleton Laboratory, UK. All reflectivity measurements were performed using time-of-flight mode, in a horizontal geometry. The neutron specular reflectivity $R$ (the ratio of the reflected beam to the incident beam intensity) was measured as a function of the momentum transfer vector $Q_{z}=4 \pi \sin \theta / \lambda$, where $\theta$ is the angle of incidence (and reflectance) and $\lambda$ is the wavelength of the neutron beam. ${ }^{47,48}$

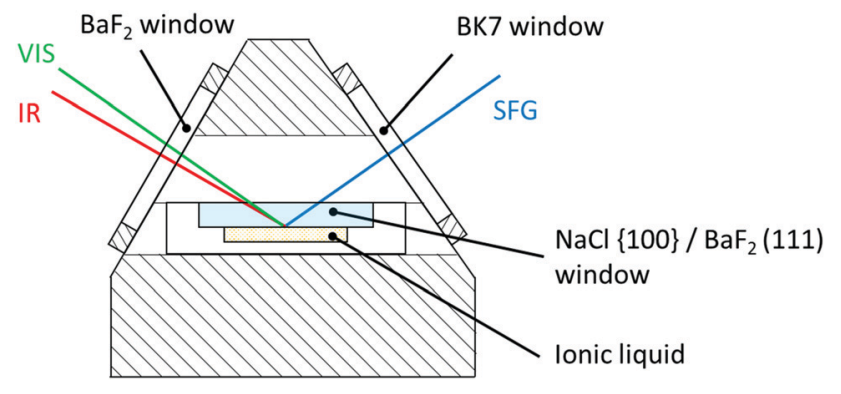

Fig. 2 Schematic of homemade SFG cell used for measurements on crystal salt surfaces and measurement geometry.
The IL reflectivity measurements were performed using a custom-made electrochemical NR cell ${ }^{31}$ ( $c f$. Section 2.3.2). The solid substrate acted as the working electrode and consisted of a layered system of $\mathrm{Ti}$ and $\mathrm{Au}$ on a Si substrate (with native oxide). The neutron beam was directed through the Si block and reflected from the gold-IL interface, with the reflected beam again traversing the block and exiting to the detector. Slits were used to define the beam size and ensure a constant beam footprint, within the area of the gold surface that was covered by IL. Prior to the IL measurements, the substrates were measured in air in order to characterise the layer parameters.

Two incident angles $\left(0.5^{\circ}\right.$ and $\left.1.8^{\circ}\right)$ were used to cover the important $Q$-range from critical edge to background (0.0065$0.22 \AA^{-1}$ ). A fixed $\mathrm{d} Q / Q$ resolution was used for all measurements (approx. $4 \%$ ). In addition, data were collected at an intermediate angle of $0.7^{\circ}$ during the equilibration time for each applied potential (30 minutes). This kinetic data was collected in event mode and divided into 10 minute intervals. An attempt to fit SLD profiles to the kinetic reflectivity curves has been made ( $c f$. ESI, $\dagger$ Fig. S8-S10), although we note the analysis of the kinetic reflectivity curves cannot provide exact models due to lower statistics and a shorter $Q$ range. However, they can indicate the dynamics and tendency of changes in the innermost layers during stabilisation times.

The scattering data were reduced in Mantid $^{49}$ using the usual procedures. The data were normalised to a transmission measurement through the silicon blocks and the two angles stitched together to form one profile. The data were fitted using the slab model based on the reflections from a stratified medium; i.e. to a model consisting of a series of layers, each with an associated thickness, roughness and scattering length density (SLD - composition). The fitting procedures were performed using the software package GenX. ${ }^{50}$ The number of SLD layers was determined as the minimum required to get precise fits of the higher $Q$ range features. The best fit was determined by using a logarithmic figure of merit (FOM). Initially, the SLDs of all layers were allowed to vary in the range determined by the maximum and minimum SLDs of the species in the sample, which are the anion and the hydrocarbon chain region of the cation respectively ( $c f$. Table 1$)$. The thickness of the layers was set to vary in the range between 2 and $20 \AA$, corresponding to the estimated thickness of the cation ring and total cation length (including the alkyl chain) which, are the shortest and longest molecular length-scales present in the IL, respectively. Initial fitting was conducted for a one-layer model and subsequent layers added if the fit improved.

Table 1 Neutron scattering length densities (SLDs) and molecular volumes $\left(V_{M}\right)$ of $\left[C_{10} C_{1} / m\right][B O B]$ ionic liquid, constituent ions and molecular segments

\begin{tabular}{lcl}
\hline Species & $\operatorname{SLD}\left(10^{-6} \AA^{-2}\right)$ & $V_{\mathrm{M}}\left(\AA^{3}\right)$ \\
\hline$\left[\mathrm{C}_{10} \mathrm{C}_{1} \mathrm{Im}\right][\mathrm{BOB}]$ & 1.53 & 582 \\
{$\left[\mathrm{C}_{10} \mathrm{C}_{1} \mathrm{Im}\right]^{+}$} & 0.33 & 321 \\
$\mathrm{C}_{1} \mathrm{Im}$ & 1.79 & 127 \\
$\mathrm{C}_{10}$ & -0.61 & 194 \\
{$[\mathrm{BOB}]^{-}$} & 3.02 & 261
\end{tabular}


The simulations were performed independently from the SFG results and no pre-defined structures were assumed. However, as the roughness of the gold electrode surface is comparable to the molecular length scales of the IL, the roughness of the near surface layers was set to match the layer thickness to account for the contribution of the gold roughness, which was estimated to be $\sim 9 \AA$ ( $c f$. ESI, $\dagger$ Table S1).

All parameters related to the substrate, i.e. the thickness, roughness and $\mathrm{SLD}$ of the $\mathrm{Au}, \mathrm{Ti}$ and $\mathrm{SiO}_{2}$ layers were obtained from the reflectivity of the blocks measured in air (cf. ESI, $\dagger$ Fig. S6) and fixed for all subsequent conditions ( $c f$. ESI, $\dagger$ Tables S2-S5). The SLD of the bulk IL was fixed for all conditions and calculated from its mass density $\left(1.17 \mathrm{~g} \mathrm{~cm}^{-3} ; c f\right.$. Table 1$){ }^{36}$ The SLDs of the ions and their molecular portions were then estimated from their calculated molar volumes ( $c f$. Table 1).

2.3.2 NR electrochemical cell and preparation of gold electrode. Details regarding the cell design have been reported previously in a related study of IL-solvent mixtures. ${ }^{31}$ However, briefly, before assembling the cell, the conductive glass (coated with a fluorine-doped tin oxide, FTO; NSG TEC A7, Pilkington, UK) used as counter electrode, as well as all the other cell components, were sonicated in $2 \%$ Hellmanex $^{\mathrm{TM}}$ III for 30 minutes, before being rinsed thoroughly with Milli-Q water and absolute ethanol, and dried with filtered $\mathrm{N}_{2}$. For the measurements presented here, a PTFE gasket of $0.5 \mathrm{~mm}$ thickness was used to separate the working (gold films) and counter electrodes. In order to minimise water contamination, the cell was assembled inside a polyethylene glove bag under a dry, nitrogen atmosphere. The IL was injected into the bottom port of the cell through PTFE tubing using a glass Luer syringe.

The thin $(\sim 120 \AA)$, amorphous gold film was prepared by electron beam evaporation (Auto 306, Edwards High Vacuum International, Wilmington, MA) atop $10 \mathrm{~mm}$ thick $(50 \times 50 \mathrm{~mm})$ polished n-type silicon(111) blocks (Sil'tronix Silicon Technologies, France). To ensure good adhesion between the Si and $\mathrm{Au}$, the block was pre-coated with a Ti adhesion layer $(\sim 55 \AA$ А; $c f$. ESI, $\dagger$ Table S1). Prior to deposition, the silicon block was cleaned in Piranha solution, rinsed with Milli-Q water and dried with filtered $\mathrm{N}_{2}$. Before conducting NR measurements in air, the coated blocks were rinsed with filtered ethanol, blow dried with nitrogen and exposed to UV/ozone for 10 minutes. Prior to all solution measurements, a short length of insulated copper wire was adhered to one corner of the gold surface using a conductive epoxy (CW400, Chemtronics), which was cured in an oven at $120{ }^{\circ} \mathrm{C}$ for $20-30$ minutes before repeating the same cleaning procedure. Once assembled and filled with IL, the cell was mounted on the beamline and the temperature maintained at $22{ }^{\circ} \mathrm{C}$. Prior to any electrical connections being made, the open circuit potential (OCP) was measured to be $-0.235 \mathrm{~V}$ across the NR cell. Electrical potentials were applied across the cell using a Metrohm Autolab (PGSTAT204) potentiostat. During all NR measurements, the applied potential and current across the cell were continually recorded and monitored $(c f$. ESI, $\dagger$ Fig. S5).

2.3.3 In situ surface charge density determination. Different potentials $(0 \mathrm{~V},-1 \mathrm{~V},+0.25 \mathrm{~V}$ and $-1.5 \mathrm{~V})$ were applied to the gold electrode interface. The surface charge density of the gold electrode was calculated for each potential by integrating the resulting electric current ( $c f$. ESI, $\dagger$ Fig. S5), which is assumed to be due to ion diffusion in the liquid. ${ }^{51}$ The surface area of the gold (working electrode) was defined by the Teflon spacer used to seal the solution and is estimated to be $17.6 \mathrm{~cm}^{2}$. The calculated surface charge densities of the gold were: $-0.6 \mu \mathrm{C} \mathrm{cm}^{-2}$ at $0 \mathrm{~V}$, $60 \mu \mathrm{C} \mathrm{cm}^{-2}$ at $+0.25 \mathrm{~V},-34 \mu \mathrm{C} \mathrm{cm}^{-2}$ at $-1 \mathrm{~V}$ and $-250 \mu \mathrm{C} \mathrm{cm}^{-2}$ at $-1.5 \mathrm{~V}$. The surface charge at $-1 \mathrm{~V}$ is comparable to that expected for mica $\left(-33 \mu \mathrm{C} \mathrm{cm}^{-2}\right)$ and also $\mathrm{NaCl}(100)$ ( $c f$. Section 2.1), whilst the surface charge density at $+0.25 \mathrm{~V}$ is close to that of the $\mathrm{BaF}_{2}(111)$ effective value. Although the surface charging mechanisms are different between metal electrodes and crystals in their origin, and there is no propensity for image charge interaction on the crystals, these effects are unlikely to affect the cation orientation and self-assembly. Application of a larger negative potential $(-1.5 \mathrm{~V})$ also allowed for the study of the IL interfacial structuring at a more highly charged surface, which is not accessible by the use of crystalline surfaces.

2.3.4 Cyclic voltammetry and surface charge determination. Cyclic voltammetry (CV) measurements were performed across the IL in the electrochemical NR cell after the reflectivity measurements using the same potentiostat ( $c f$. Section 2.3.2). To establish that no faradaic events occurred within the experimental potential window studied, as well as determine the reversibility of the charge transfer, the potential was cycled between $-1.5 \mathrm{~V}$ and $+0.25 \mathrm{~V}$ several times at different rates ( 6 and $10 \mathrm{mV} \mathrm{s}^{-1}$ ) and the current response monitored. Representative CVs for each rate are shown in ESI $\dagger$ (cf. Fig. S1).

\section{Results and discussion}

\subsection{Sum-frequency generation spectroscopy}

Fig. 3 and 4 show the SFG spectra of each IL at $\mathrm{BaF}_{2}(111)$ and $\mathrm{NaCl}(100)$, respectively. SSP and PPP refer to two different polarisation combinations of the signal and the two incident beams. From analysis of the relative intensities of a given peak in the two polarisation combinations, conclusions can be drawn about the orientation of the vibrational mode. ${ }^{52}$ The $[\mathrm{BOB}]^{-}$ anion has an inversion-symmetry and shows a strong quadrupole moment. ${ }^{53}$ The quadrupole contribution cannot therefore be excluded for the $[\mathrm{BOB}]^{-}$anion signal and in this case SFG is no longer interface sensitive. We have thus focused on the imidazolium cations to avoid complication of the SFG analysis.

Aliphatic C-H stretching modes appeared at $2800-3000 \mathrm{~cm}^{-1}$. Peaks at $2850 \mathrm{~cm}^{-1}, 2875 \mathrm{~cm}^{-1}$ and $2945 \mathrm{~cm}^{-1}$ were observed in the SSP spectra and can be attributed to $\mathrm{CH}_{2}$-ss (symmetric stretching vibrational mode), $\mathrm{CH}_{3}$-ss and the Fermi resonance of $\mathrm{CH}_{3}$-ss, respectively. Peaks at $2890 \mathrm{~cm}^{-1}$ and $2960 \mathrm{~cm}^{-1}$, which are attributed to $\alpha-\mathrm{CH}_{2}$-as (antisymmetric stretching vibrational mode of methylene next to methyl group) and $\mathrm{CH}_{3}$-as (asymmetric stretching vibrational mode), respectively, were observed in the PPP spectra. ${ }^{54,55}$ Aromatic C-H stretching modes were observed at $3000-3250 \mathrm{~cm}^{-1}$. The peaks at $3127 \mathrm{~cm}^{-1}\left[\mathrm{C}_{(2)} \mathrm{H}\right], 3153 \mathrm{~cm}^{-1}$ $\left[\mathrm{HC}_{(4)}-\mathrm{C}_{(5)} \mathrm{H}-\mathrm{as}\right]$, and $3174 \mathrm{~cm}^{-1}\left[\mathrm{HC}_{(4)}-\mathrm{C}_{(5)} \mathrm{H}-\mathrm{ss}\right]$ are assumed to be from the $\mathrm{C}-\mathrm{H}$ normal mode vibrations of the hydrogen attached 
(a)
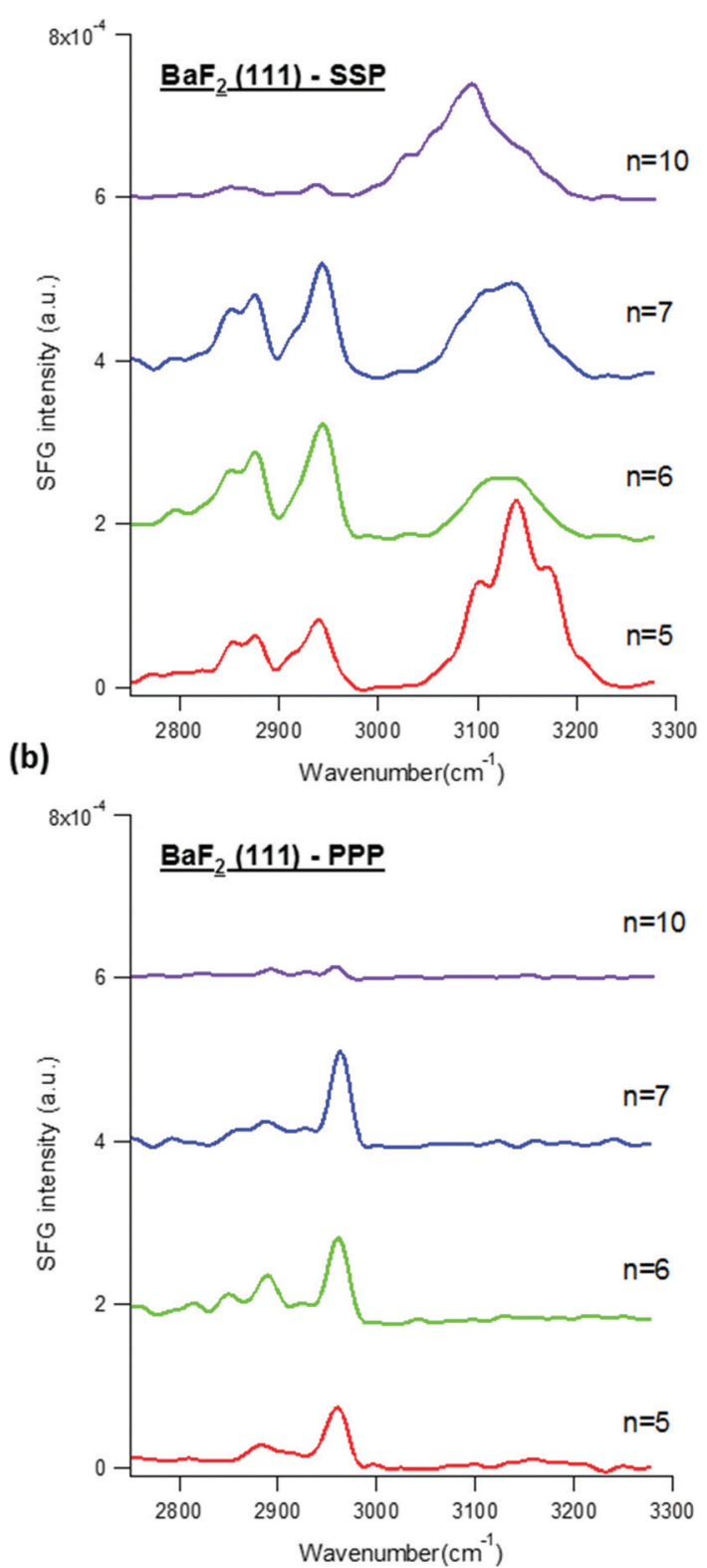

Fig. 3 SFG spectra of $C_{5} C_{1} 1 m[B O B], C_{6} C_{1} 1 m[B O B], C_{7} C_{1} 1 m[B O B]$ and $\mathrm{C}_{10} \mathrm{C}_{1} \operatorname{lm}[\mathrm{BOB}]$ at positively charged $\mathrm{BaF}_{2}(111)$ with (a) SSP and (b) PPP polarisations.

directly to the cation's imidazolium ring. In this wavenumber range, some Fermi resonance peaks were also present, ${ }^{29,30}$ due to overtones of the imidazolium ring and combination of in-plane ring modes, making quantitative and accurate orientational angle analysis complex. However, the presence of an SFG signal is a clear indication of a non-isotropic net orientation.

Conversely, $\left[\mathrm{C}_{10} \mathrm{C}_{1} \mathrm{Im}\right][\mathrm{BOB}]$ showed notably weak peaks at both the $\mathrm{NaCl}(100)$ and $\mathrm{BaF}_{2}(111)$ surfaces, suggesting a cancelling out of the SFG signal from the cation alkyl chains. Two possible near surface structures could lead to such signal cancelling: a bilayer structure, where all the cation decyl chains from the bilayer lower and upper monolayer leaflets orientate (a)
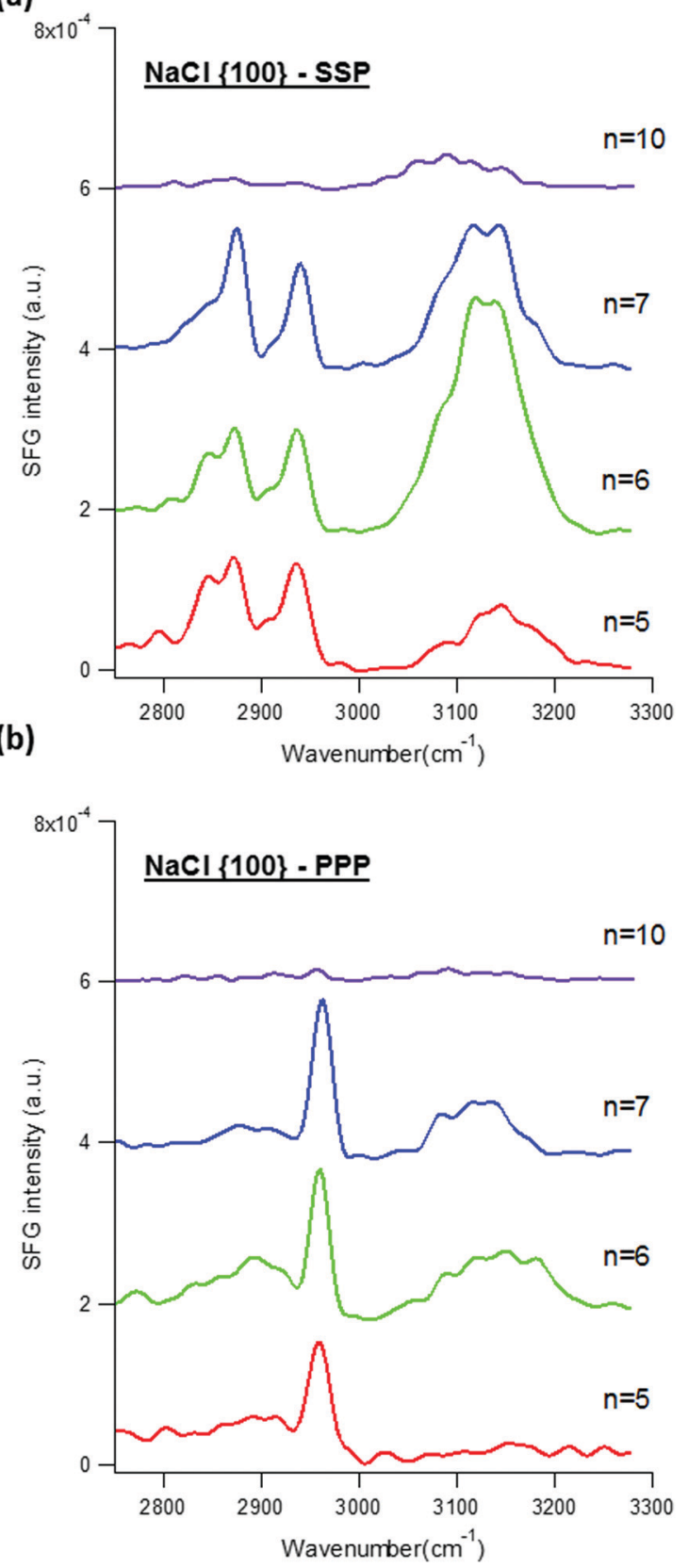

Fig. 4 SFG spectra of $C_{5} C_{1} \operatorname{lm}[B O B], C_{6} C_{1} \operatorname{lm}[B O B], C_{7} C_{1} \operatorname{lm}[B O B]$ and $\mathrm{C}_{10} \mathrm{C}_{1} \operatorname{lm}[\mathrm{BOB}]$ at negatively charged $\mathrm{NaCl}(100)$ with (a) SSP and (b) PPP polarisations.

towards each other in the surface normal, or a completely isotropic and randomly oriented layer. (Note that a random orientation between 0 and $180^{\circ}$ will lead to SFG signal with polarisation dependency corresponding to the so-called "magic angle". $\left.{ }^{56,57}\right)$ On the other hand, $\left[\mathrm{C}_{n} \mathrm{C}_{1} \mathrm{Im}\right][\mathrm{BOB}](n=5-7)$ showed strong aliphatic $\mathrm{C}-\mathrm{H}$ peaks deriving from the cation alkyl chains, indicating the short alkyl chain cations do not form bilayers (see ESI, $\dagger$ Fig. S11 for further analysis). In the PPP spectra at both $\mathrm{NaCl}(100)$ and $\mathrm{BaF}_{2}(111)$ surfaces, a peak at $2890 \mathrm{~cm}^{-1}$ was observed, which is attributed to $\alpha-\mathrm{CH}_{2}$-a. This mode is not strongly active in SFG, therefore accounting for the 
relatively weak signal, ${ }^{55}$ and suggests that gauche defects are introduced to the methylene group closest to the terminal methyl group of the alkyl chain. ${ }^{58}$ In turn, this indicates that the layer is not a perfectly ordered self-assembly structure with parallel packed chains, as might be observed for a conventional surfactant or thiol self-assembly monolayer (SAM) ${ }^{59}$

Consideration of the aromatic $\mathrm{C}-\mathrm{H}$ region reveals that all the ILs exhibited prominent aromatic $\mathrm{C}-\mathrm{H}$ peaks at the $\mathrm{BaF}_{2}(111)$ surface under SSP polarisation, but no peaks with PPP polarisation. The molecular orientation analysis reveals that the imidazolium ring adopts a more perpendicular orientation at the $\mathrm{BaF}_{2}(111)$ surface ( $c f$. ESI, $\dagger$ Fig. S2 and S3). Conversely, at the $\mathrm{NaCl}(100)$ surface all the ILs exhibited aromatic $\mathrm{C}-\mathrm{H}$ peaks with both SSP and PPP polarisations. As noted above, a detailed analysis of the orientational angle of the imidazolium ring is difficult due to interference with the Fermi resonance of ring stretching modes. ${ }^{29,30}$ However, the presence of the $\mathrm{C}-\mathrm{H}$ peaks indicates that the imidazolium ring has a tilted orientation. Together these observations suggest the imidazolium ring is attracted to negatively charged $\mathrm{NaCl}(100)$ surface and has a tendency to adopt a parallel orientation to the surface. Conversely, on the positively charged $\mathrm{BaF}_{2}(111)$ surface, the imidazolium ring tends towards a more perpendicular orientation, leading to a larger distance of the positive charge from the surface, as well as allowing more anions to come close to the surface, reflecting a repulsive interaction between the ring and the surface. A similar tendency has been reported in the electrochemical SFG measurements of halogenated ILs performed by Baldelli et al. ${ }^{23,24}$ Moreover, the SSP spectra of $\left[\mathrm{C}_{10} \mathrm{C}_{1} \mathrm{Im}\right][\mathrm{BOB}]$ at both the $\mathrm{NaCl}(100)$ and $\mathrm{BaF}_{2}(111)$ surfaces, show a larger redshift (i.e. shift toward lower wavenumbers) of the aromatic peaks than observed for the short alkyl chain ILs $\left[\mathrm{C}_{n} \mathrm{C}_{1} \mathrm{Im}\right][\mathrm{BOB}]$, where $n=5-7$.

Previous IR and Raman spectroscopy studies have demonstrated that the peak position of $\mathrm{C}_{(2)}-\mathrm{H}$, which is the most interactive with the anion, can undergo a redshift with increasing alkyl chain length, and attributed this to improper hydrogen bonds caused by an inductive effect, whereby the donor bonds are less polar and electron poor, and the bond acceptor is not sufficiently strong for hydrogen bonding. ${ }^{60,61}$ This tendency was observed even in the presence of Fermi resonances and overtones. ${ }^{62}$ Here, we similarly observe a redshift in the IR spectra ( $c f$. ESI, $\dagger$ Fig. S4). However, the redshift with increasing chain length is less remarkable (only a few $\mathrm{cm}^{-1}$ ) than observed in the SFG spectra.

A number of other studies have also reported that the peak position of $\mathrm{C}_{(2)}-\mathrm{H}$ can undergo a redshift when the cation interacts with more basic and coordinating anions, such as $\mathrm{N}(\mathrm{CN})_{2}$ and halogens. ${ }^{30,63-65}$ This redshift is hypothesised to occur due to an increase in strength of hydrogen bonding between cation and anion, especially at the cation $\mathrm{C}_{(2)}-\mathrm{H}$ position, and can cause a redshift up to $100 \mathrm{~cm}^{-1}$. In light of these previous studies, the redshift in SFG spectra presented here thus indicates that the interaction between cation and anion and/or the surface is much stronger than cation-anion interaction in bulk liquid. A possible explanation of the prominent redshift in the case of $\left[\mathrm{C}_{10} \mathrm{C}_{1} \mathrm{Im}\right]$ [BOB] is that the bilayer formation by the alkyl chains of cations leads to a more distinct segregation between the polar and nonpolar moieties, and hence, in the innermost layer, the cation and anion polar parts are closely packed.

\subsection{Neutron reflectivity}

NR is a powerful tool that can be used to characterise the interfacial structures of ILs with sub-nanometer resolution in the surface normal direction. ${ }^{13,31,32,34,66}$ It is clear from the SFG results presented above that the structuring of the cation alkyl chains at charged interfaces is notably different for the longest chain $\left[\mathrm{C}_{n} \mathrm{C}_{1} \mathrm{Im}\right][\mathrm{BOB}]$ IL $(n=10)$. Two possible hypothesises for this observation (discussed above) are: (1) the cation decyl chains form a well-ordered bilayer structure, or (2) they form a completely isotropic and randomly orientated layer, which is unlikely, but cannot be ruled out from SFG measurements alone. For this reason, complementary NR measurements were conducted to characterise the out-of-plane structuring of the $\mathrm{IL}$ $\left[\mathrm{C}_{10} \mathrm{C}_{1} \mathrm{Im}\right][\mathrm{BOB}]$ at different surface potentials. The surface potential was controlled in situ through use of an electrochemical cell. ${ }^{36}$ This approach is preferable for NR measurements, since there are a limited number of substrates that lend themselves to NR measurement in terms of scattering length density (SLD) and roughness. By the same token, non-resonant background signals render SFG measurement next to impossible on gold electrodes.

3.2.1 Equilibrium NR measurements. Fig. 5a shows the reflectivity curves and corresponding model fits to the data for each potential studied. Clear Kiessig fringes are seen in the reflectance due to contrast in SLD between the gold electrode and the IL. Theoretical fits to the data reveal that in the immediate vicinity of the surface, the obtained SLD profiles cannot be explained by the presence of bulk (isotropic) IL. Thus, the possible hypothesis of an isotropic interface, as discussed above, can be deemed unlikely. Instead, for all potentials a non-monotonic SLD profile was found to best fit the reflectivities, by dividing the interfacial region into layers of varying $S L D$, thickness and roughness ( $c f$. Fig. 5b). In the SLD profiles it is difficult to distinguish a distinct first layer due to the contribution of the gold roughness, which is comparable in size to the molecular dimensions of the IL. However, for all potentials, a deep minimum in the SLD profile next to the gold interface is indicative of a distinct, lower SLD layer which is therefore likely to be composed of cation alkyl chains (cf. Table 1).

For $0 \mathrm{~V}$, the trough of this minimum is notably closer to the gold interface, suggesting the cation alkyl chains structure directly at the gold interface ( $c f$. ESI, $\dagger$ Fig. S7 and Table S2). However, for non-zero surface potentials, a clear shift of the minimum away from the gold interface is apparent, in particular for the positive potential $(+0.25 \mathrm{~V})$, pointing towards the presence of a (buried) first layer, the thickness of which is larger for the positive potential. The exact origin of this thickness change is difficult to determine, however it is not inconsistent with the SFG findings presented above ( $c f$. Section 3.1), whereby the orientation of the cation rings is shown to become more perpendicular with respect to the surface normal for the positive surface potential. However, an increased thickness would also be commensurate with an increase in anion density 


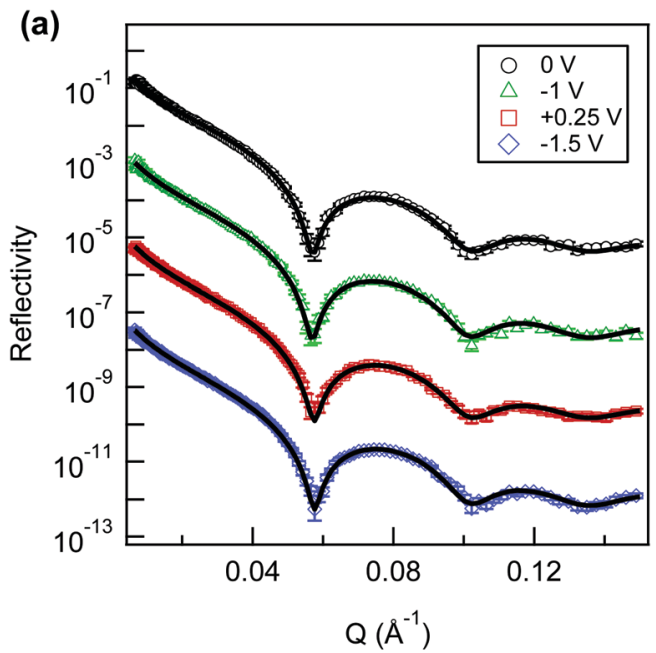

(b)

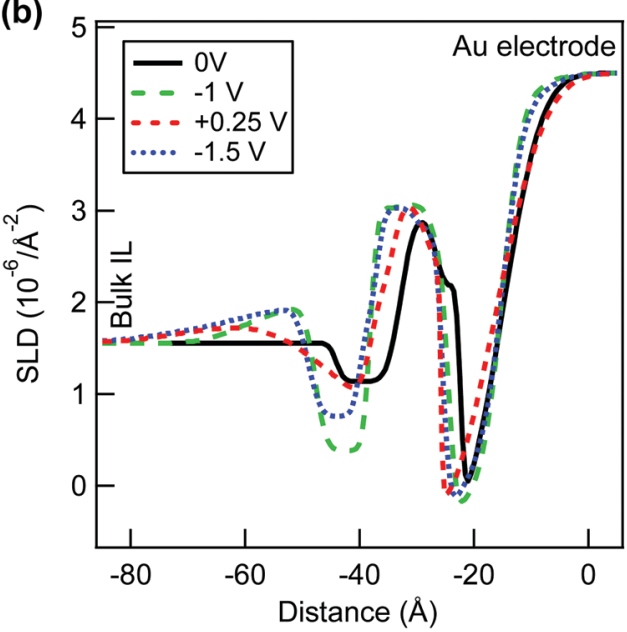

Fig. 5 (a) Neutron reflectivity curves for $\left[\mathrm{C}_{10} \mathrm{C}_{1} \mathrm{Im}\right][\mathrm{BOB}]$ at a gold electrode for different applied potentials. The symbols represent experimental data, while the solid lines represent fitted scattering length density (SLD) model fits to the data. (b) Corresponding SLD profiles.

at the interface, whose size is larger than that of the imidazolium ring, and can be expected to be driven towards the electrode interface to counter the positive surface potential. For the non-zero surface potentials, the thickness of the initial minimum at the gold interface is also notably larger, suggesting the transition from a single alkyl chain layer to that of an interdigitated bilayer structure.

Further away from the gold interface, two more distinct regions of higher and lower SLD than that of the bulk IL, respectively, can be observed each of the different potential SLD profiles. At $0 \mathrm{~V}$, these layers are located notably closer to the gold interface, due to the absence of an initial cation ring layer. For the negative potentials $(-1 \mathrm{~V}$ and $-1.5 \mathrm{~V})$, the high SLD region, which can be attributed to a high density of anions, extends further into the bulk, suggesting a larger counterion region. For $-1.5 \mathrm{~V}$, the second low SLD region (or minima in the SLD profile) is also of noticeably lower SLD and broader, suggestive of a subsequent alkyl chain bilayer.

Schematic interfacial structures based on the model parameters used to fit the reflectivities for $\left[\mathrm{C}_{10} \mathrm{C}_{1} \mathrm{Im}\right][\mathrm{BOB}]$ at different surface potentials ( $c f$. ESI, $\dagger$ Tables S3-S5) are shown in Fig. 6. In addition, a schematic for $0 \mathrm{~V}$ is provided in the ESI $\dagger$ (cf. Fig. S7). These structures are consistent with the SLD profiles in Fig. 5b, but cannot be deemed unique solutions. Nonetheless, for all conditions where the gold electrode bears a notable charge, i.e. $\neq 0 \mathrm{~V}$, the SLD profile and model parameters clearly advocate that the $\left[\mathrm{C}_{10} \mathrm{C}_{1} \mathrm{Im}\right]$ cations form a bilayer-like structure at the gold interface, whereby the methylimidazolium rings form a distinct inner layer at the electrode interface. For the larger negative surface potential $(-1.5 \mathrm{~V})$, this initial bilayer is followed by a similar, but more diffuse, bilayer-like structure atop it. Clear changes in the thickness of the inner IL layer at the gold electrode with potential also point towards a significant reorientation of the imidazolium ring with respect to the gold interface, depending on the surface charge bias. However, we acknowledge that a larger thickness observed at $+0.25 \mathrm{~V}$ could also be attributable to small population of anions at the gold interface.

3.2.2 Kinetic NR measurements. Reflectivity was collected during the electrical stabilisation period (of 30 minutes) after each potential, ( $c f$. ESI, $\dagger$ Fig. S8-S10). SLD fits to the data, when divided into three successive $600 \mathrm{~s}$ intervals, reveal the time dependence of the response, and give insight into the re-arrangement of ion layers and imidazolium ring orientation changes during surface
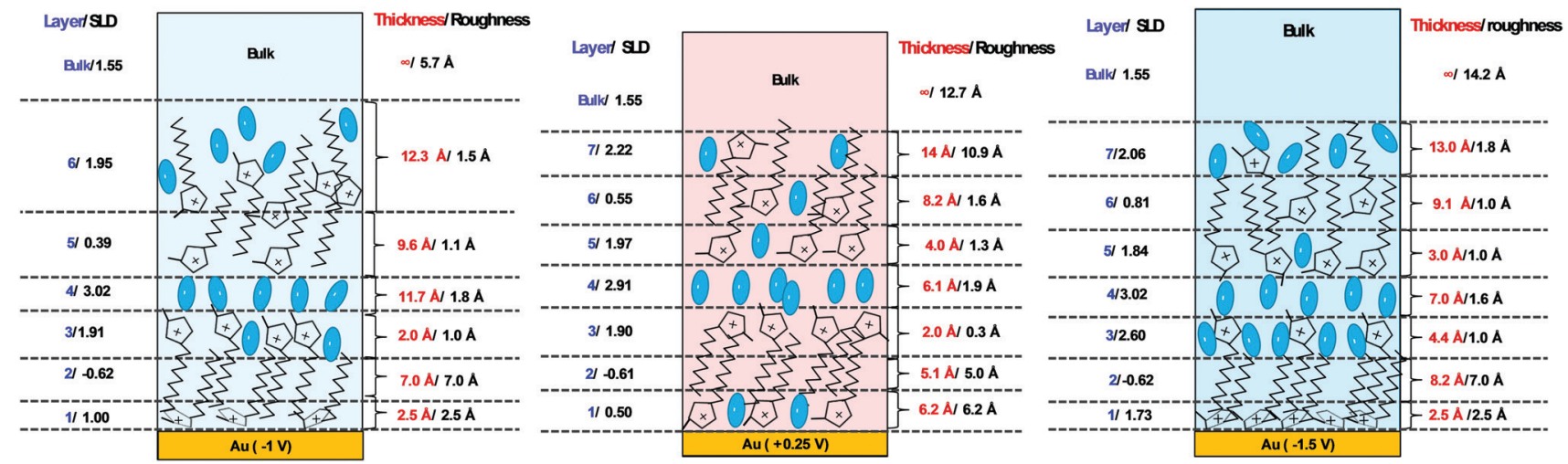

Fig. 6 Schematic illustrations of the interfacial structures of the ionic liquid $\left[\mathrm{C}_{10} \mathrm{C}_{1} \mathrm{Im}\right][\mathrm{BOB}]$ at different applied surface potentials $(-1,+0.25,-1.5 \mathrm{~V})$ in experimental order after initially applying $0 \mathrm{~V}$. The schematic model for $0 \mathrm{~V}$ is shown in the ESI† (cf. Fig. S7). 
potential equilibration. In each case, the final time-slice SLD profile is similar to that obtained for the long, post-equilibration scans shown in Fig. 5b, indicating that the allowed equilibration period was sufficient.

After applying $-1 \mathrm{~V}$ from $0 \mathrm{~V}$, the kinetic SLD profiles $(c f$. ESI; $\dagger$ Fig. S8b) suggest that a re-arrangement in the innermost layer occurs, due to imidazolium rings of the cations orienting towards the surface and the formation of three distinct cation rich layers, followed by a layer of anions. Subsequently, after applying $+0.25 \mathrm{~V}$ ( $c f$. ESI; $\dagger$ Fig. S9b), the kinetic SLD profiles imply that the imidazolium rings are repelled from the surface, leading to the formation of a more diffuse structuring, without distinct cation-anion layering. Instead, the cation decyl chains remain close to the surface, with anions accommodated amongst them. Within the final time interval, a more distinct anion layer is then formed further away from the gold electrode atop the cation layer. After $-1.5 \mathrm{~V}$ was applied ( $c f$. ESI; $\dagger$ Fig. S10a), a shift in the kinetic reflectivity curves towards higher $Q$ values was observed. In the first time interval (the initial state), the kinetic SLD profile ( $c f$. ESI; $\dagger c f$. Fig. S10b) indicates that anions are repelled from the surface, whilst the cations are attracted and form a bilayer.

\subsection{Interfacial structure of the ionic liquids at positively/negatively charged surface}

The SFG spectra for all the ILs studied indicate that the cation alkyl chain length has a strong influence on interfacial structures of imidazolium-based ILs, even at charged interfaces. That is to say, the longer alkyl chain $\left(\left[\mathrm{C}_{10} \mathrm{C}_{1} \mathrm{Im}\right][\mathrm{BOB}]\right)$ cation forms a bilayer of alkyl chains at both negatively and positively charged surfaces, whilst its shorter alkyl chain $\left(\left[\mathrm{C}_{n} \mathrm{C}_{1} \mathrm{Im}\right][\mathrm{BOB}] ; n=5-7\right)$ analogues do not. The formation of a bilayer of alkyl chains in the case of $\left[\mathrm{C}_{10} \mathrm{C}_{1} \mathrm{Im}\right][\mathrm{BOB}]$ is also confirmed by the results of NR measurement. The electric double layers formed in pure ILs can be very different from those formed in conventional dilute electrolyte solutions. Charged moieties of cation and anion are stabilised energetically if surrounded by opposite charges or by polar materials or polarisable materials. Thus, the self-assembly of ILs, which are composed of only cations and anions, is primarily driven by Coulombic interactions, resulting in the formation of alternating layers of cations and anions. ${ }^{67-70}$ Such multi-layering has also been suggested by molecular dynamic (MD) simulations, ${ }^{71-73}$ and is in better agreement with the experimental results ${ }^{24,74}$ than models applicable to dilute electrolyte solutions.

Based on this Coulombically alternating multilayer formation, the interfacial structure of the ILs in this study can be explained as follows. The first layer is occupied by counter ions, as indicated by the NR measurements, and the orientation of the imidazolium ring is determined by the surface charge, as observed in the SFG spectra. Bazant et al. ${ }^{69}$ have previously suggested the surface charge tends to be overscreened by the first layer within this surface charge density range. In the case of shorter alkyl chains ( $n=5-7$ ), the second layer consists of co-ions, of opposite charge to the first layer and like charge to the surface charge, based on Coulombic interaction. The third and fourth layers are analogous, but with a decreasing degree of order, reflecting screening of the surface charge. Interestingly, however, a simple Coulombic alternating multilayer model is no longer applicable when the alkyl chain is sufficiently long (in this case for $n=10$ ). Instead, in the case of a cation bilayer, the second layer is populated by the second monolayer leaflet, which is then followed by a subsequent third layer of co-ions. Thus, when the alkyl chain of cation is long enough, the IL organises into polar and nonpolar domains. ${ }^{75}$ Some simulation results have demonstrated that imidazolium ILs can undergo spontaneous micro-phase separation, and this effect becomes dominant when increasing the alkyl chain length. ${ }^{76,77}$ This segregation into polar and nonpolar domains is consistent with a redshift of the aromatic $\mathrm{C}-\mathrm{H}$ peaks in the SFG spectra, and therefore suggests that the $\mathrm{C}_{(2)}-\mathrm{H}$ group of the cation imidazolium ring comes closer to the anion and the negatively charged surface than it does in the shorterchain analogues. The larger nonpolar moieties in the case of $\left[\mathrm{C}_{10} \mathrm{C}_{1} \mathrm{Im}\right]$ would lead to a locally reduced dielectric environment for the ions if there were mixing of the decyl chains and ions; thus, the system self-assembles to optimise both the interactions with the surface and locally between ions. This form of molecular ordering bears similarity to self-assembly of amphiphiles in water, but with one important difference - the latter process is driven entropically, and it is hard to envisage a driver equivalent to the hydrophobic effect in a pure IL. ${ }^{78}$ For example, in the case of protic ILs (PILs) it has been shown that the self-assembly of ionic amphiphiles is controlled rather by specific, short-range solvation and H-bonding interactions between the surfactant and the particular PIL. ${ }^{79}$

Interestingly, despite the strong dependence of the interfacial structure monolayer/bilayer formation on the alkyl chain length, irrespective of surface charge, the SFG spectra also show apparent differences in the tendency of the imidazolium ring to orient at negatively and positively charged surfaces. In addition to revealing differences in self-assembly behaviour (a largely charge independent phenomenon), the SFG spectra also indicate differences in the orientation behaviour of the imidazolium ring in response to surface charge. Whilst the imidazolium ring has a tilted orientation at the negatively charged surface, the ring orients perpendicular to the positively charged surface, increasing the distance between the surface and cation charges. It should be remembered that the SLD fits to the NR do not directly provide proof of the presence of the ring at the surface (due to the potential contributions of several species). Rather the SLD values and thickness together indicate that indeed the cation ring is closest to the surface, and that it adopts a more perpendicular orientation at the electrode surface when a positive potential is applied (i.e., the innermost layer thickness of $5 \AA$ corresponds well with the estimated length of the cation ring). We note that recent computations suggest that the adsorption of alkyl decorated imidazolium ILs to gold is a subtle process, with contributions involving cation $\cdots \pi$ stacking and anion-induced anchor-assisted H-bonds. Although the predominance of these various contributions is expected to vary depending on the polarity of the IL anion and was not investigated for different surface potentials. ${ }^{80}$

At the negative potential $(-1 \mathrm{~V})$, the much thinner surface layer $(2.5 \AA)$ is more comparable with the ring thickness and 
thus also suggests a parallel orientation of the cation ring. Both of these observations are entirely consistent with the SFG data on surfaces of comparable charge. Upon further increasing the negative surface potential to $-1.5 \mathrm{~V}$, not typically accessible without an electrical bias, it is found that the bilayer structure of the IL extends further into the bulk, suggesting not only the bias, but also the magnitude of the surface potential can be used to control the interfacial structuring of imidazoliumbased ILs. Furthermore, this indicates that the surface charge is too high to be compensated by a single counterion layer, which is the case for the other smaller potentials studied. In a "typical" multilayering IL system, such as that displayed by the shorter alkyl chains $(n=5-7)$, the ionic species forming each layer are dependent on surface charge, i.e., the first layer is occupied by the counterion to the surface charge, while subsequent layers consist of alternating layers of predominantly co-ions and counterions. In the case of long alkyl chain $(n=10)$, however, although the first layer is responsive to the surface charge, the cations form a bilayer structure at the surface for both negatively and positively charged surfaces. The NR measurements indicate that the thickness of each component varies with surface charge. Such thickness variations are likely to be largely due to changes in the density and degree of ordering of the cations in the first layer. The degree of ordering of cation layers also determines the charge density of the layer.

It has been previously shown that the friction reduction properties of boundary films are largely dependent on surface density and degree of ordering. ${ }^{81,82}$ Moreover, as mentioned in the introduction, robust bilayer structures have previously been shown to prevent breakdown of boundary films and lead to good lubricity. In light of these observations, bilayer forming IL systems would allow for the tuning of both thickness and degree of ordering through the application of electric potentials, whilst retaining their lubricating bilayer structure. This presents a promising mechanism for friction control.

\section{Conclusions}

While our knowledge of ILs containing fluorinated ILs is fast expanding, more remains to be done on potentially more applicable materials, for example with orthoborate anions, as used here. The results bring a new perspective to the structuring of ILs at charged interfaces, and the sensitivity of the charge to the sign and magnitude of the electrical potential. By combining NR and SFG, we demonstrate how the composition of the various layers, as well as orientations of the various species in response to changes in surface charge. Both the orientation of the imidazolium ring, and the alkyl regions of the cation can be rather sensitively targeted using both techniques.

From the results obtained, it can be understood that the first layer clearly responds to surface charge, regardless of the alkyl chain length. For the shorter $(n=5-7)$ alkyl chain cations, the second layer is then occupied by co-ions to compensate the ion charge of inner counterion layer. In contrast, for the longer $(n=10)$ alkyl chain cation, the second layer is instead of charge compensation by co-ions, the cations form a bilayer structure.
Together, these results demonstrate that if the alkyl chain is sufficiently long, the non-polar aggregation of alkyl chains will supersede the charge compensation of ions, resulting in the formation of cation bilayers. Here, whilst surface charge drives the formation of electric double layer of ILs at the surface, the structure of the more extended layers is strongly dependent on alkyl-chain length of cation. This is rather evocative of selfassembly in surfactant systems, and confirms the observation of bilayers in ILs with surfactant-like cations (where the positive charge is relatively well defined analogous to a surfactant headgroup, with a long hydrocarbon tail). It should be remembered that it is the hydrophobic effect which leads to such self-assembly, and strictly this mechanism cannot operate in an IL. Thus, the role of interfacial water cannot be completely ruled out as a contributor, even under the dry conditions used here. Alternatively, the bilayer structure maybe due to a templating of the self-assembly tendency (driven by the expulsion of nonpolar regions from polar charged regions) by the surface. Future work will thus target: (i) the role of water at the interface, and (ii) the effect of alkylation on the orthoborate anion - which will allow the anion also to contribute to any self-assembly behaviour.

Finally, the preservation of the bilayer structure, even for very different surface charges means that the essential boundary lubrication properties can be maintained, while the composition of the near surface layer can be tuned to moderate properties in interfacial contact.

\section{Conflicts of interest}

There are no conflicts to declare.

\section{Acknowledgements}

We acknowledge the Knut and Alice Wallenberg Foundation (Project No. KAW2012.0078), the Swedish Research Council (Project No. 2014-4694) and the Swedish Foundation for Strategic Research (Project No. EM16-0013) for their financial support. We gratefully acknowledge the Science and Technology Facilities Council (STFC) for access to neutron beamtime at ISIS (Experiment RB1810114; 10.5286/ISIS.E.RB1810114), the provision of sample preparation and INTER facilities.

\section{References}

1 P. Wasserscheid and T. Welton, Ionic liquids in synthesis, John Wiley \& Sons, 2008.

2 S. Glavatskih and E. Höglund, Tribol. Int., 2008, 41, 934-939.

3 J. Sweeney, F. Hausen, R. Hayes, G. B. Webber, F. Endres, M. W. Rutland, R. Bennewitz and R. Atkin, Phys. Rev. Lett., 2012, 109, 155502.

4 Y. Kondo, S. Yagi, T. Koyama, R. Tsuboi and S. Sasaki, Proc. Inst. Mech. Eng., Part J, 2012, 226, 991-1006.

5 F. U. Shah, S. Glavatskih, D. R. MacFarlane, A. Somers, M. Forsyth and O. N. Antzutkin, Phys. Chem. Chem. Phys., 2011, 13, 12865-12873. 
6 F. U. Shah, S. Glavatskih and O. N. Antzutkin, Tribol. Lett., 2013, 51, 281-301.

7 T. Welton, Chem. Rev., 1999, 99, 2071-2084.

8 S. Campen, J. Green, G. Lamb and H. Spikes, Tribol. Lett., 2015, 57, 18.

9 H. Spikes, Tribol. Lett., 2015, 60, 5.

10 L. Kong, W. Huang and X. Wang, J. Phys. D: Appl. Phys., 2016, 49, 225301.

11 H. Li, R. J. Wood, F. Endres and R. Atkin, J. Phys.: Condens. Matter, 2014, 26, 284115.

12 S. Perkin, L. Crowhurst, H. Niedermeyer, T. Welton, A. M. Smith and N. N. Gosvami, Chem. Commun., 2011, 47, 6572-6574.

13 L. R. Griffin, K. L. Browning, S. M. Clarke, A. M. Smith, S. Perkin, M. W. A. Skoda and S. E. Norman, Phys. Chem. Chem. Phys., 2017, 19, 297-304.

14 M. Yao, M. Fan, Y. Liang, F. Zhou and Y. Xia, Wear, 2010, 268, 67-71.

15 X. Liu, F. Zhou, Y. Liang and W. Liu, Wear, 2006, 261, 1174-1179.

16 K. Boschkova, A. Feiler, B. Kronberg and J. Stålgren, Langmuir, 2002, 18, 7930-7935.

17 A. M. Smith, M. A. Parkes and S. Perkin, J. Phys. Chem. Lett., 2014, 5, 4032-4037.

18 W. H. Briscoe, S. Titmuss, F. Tiberg, R. K. Thomas, D. J. McGillivray and J. Klein, Nature, 2006, 444, 191-194.

19 P. K. Cooper, C. J. Wear, H. Li and R. Atkin, ACS Sustainable Chem. Eng., 2017, 5, 11737-11743.

20 M. Radiom, P. Pedraz, G. Pilkington, P. Rohlmann, S. Glavatskih and M. W. Rutland, Colloids Interfaces, 2018, 2, 60.

21 H. Zhou, M. Rouha, G. Feng, S. S. Lee, H. Docherty, P. Fenter, P. T. Cummings, P. F. Fulvio, S. Dai, J. McDonough, V. Presser and Y. Gogotsi, ACS Nano, 2012, 6, 9818-9827.

22 M. L. Schlegel, K. L. Nagy, P. Fenter, L. Cheng, N. C. Sturchio and S. D. Jacobsen, Geochim. Cosmochim. Acta, 2006, 70, 3549-3565.

23 S. Baldelli, Acc. Chem. Res., 2008, 41, 421-431.

24 C. Aliaga, C. S. Santos and S. Baldelli, Phys. Chem. Chem. Phys., 2007, 9, 3683-3700.

25 S. Watanabe, M. Nakano, K. Miyake, R. Tsuboi and S. Sasaki, Langmuir, 2014, 30, 8078-8084.

26 H. Li, F. Endres and R. Atkin, Phys. Chem. Chem. Phys., 2013, 15, 14624-14633.

27 C. Y. Peñalber and S. Baldelli, J. Phys. Chem. Lett., 2012, 3, 844-847.

28 C. Y. Peñalber, G. A. Baker and S. Baldelli, J. Phys. Chem. B, 2013, 117, 5939-5949.

29 J.-C. Lassègues, J. Grondin, D. Cavagnat and P. Johansson, J. Phys. Chem. A, 2009, 113, 6419-6421.

30 J. Grondin, J. C. Lassègues, D. Cavagnat, T. Buffeteau, P. Johansson and R. Holomb, J. Raman Spectrosc., 2011, 42, 733-743.

31 G. A. Pilkington, K. Harris, E. Bergendal, A. B. Reddy, G. K. Palsson, A. Vorobiev, O. N. Antzutkin, S. Glavatskih and M. W. Rutland, J. Chem. Phys., 2018, 148, 193806.

32 Y. Lauw, M. D. Horne, T. Rodopoulos, V. Lockett, B. Akgun, W. A. Hamilton and A. R. J. Nelson, Langmuir, 2012, 28, 7374-7381.
33 G. A. Pilkington, A. Oleshkevych, P. P. Carrasco, S. Watanabe, M. Radiom, A. B. Reddy, A. Vorobiev, S. Glavatskih and M. W. Rutland, Tribotronic structuring and friction of a non-halogenated ionic liquid in a polar solvent: Effect of concentration, in preparation.

34 N. Nishi, J. Uchiyashiki, Y. Ikeda, S. Katakura, T. Oda, M. Hino and N. L. Yamada, J. Phys. Chem. C, 2019, 123, 9223-9230.

35 A. Filippov, N. Azancheev, A. Gibaydullin, S. Bhattacharyya, O. N. Antzutkin and F. U. Shah, Magn. Reson. Chem., 2018, 56, 113-119.

36 P. Rohlmann, M. R. Shimpi, M. W. Rutland, O. Antzukin and S. Glavatskih, Tuning the transport properties of tribologically relevant ionic liquids, in preparation.

37 J. Vogt, J. Henning and H. Weiss, Surf. Sci., 2005, 578, 57-70. 38 N. Sieffert and G. Wipff, J. Phys. Chem. B, 2007, 111, 7253-7266. 39 X. Zhu, H. Suhr and Y. Shen, Phys. Rev. B: Condens. Matter Mater. Phys., 1987, 35, 3047.

40 C. Hirose, N. Akamatsu and K. Domen, J. Chem. Phys., 1992, 96, 997-1004.

41 R. Ward, P. Davies and C. Bain, J. Phys. Chem., 1993, 97, 7141-7143.

42 J. Hedberg, J. Henriquez, S. Baldelli, C. M. Johnson and C. Leygraf, J. Phys. Chem. C, 2008, 113, 2088-2095.

43 H.-W. Cheng, P. Stock, B. Moeremans, T. Baimpos, X. Banquy, F. U. Renner and M. Valtiner, Adv. Mater. Interfaces, 2015, 2, 1500159.

44 G. Feng, X. Jiang, R. Qiao and A. A. Kornyshev, ACS Nano, 2014, 8, 11685-11694.

45 S. Bi, R. Wang, S. Liu, J. Yan, B. Mao, A. A. Kornyshev and G. Feng, Nat. Commun., 2018, 9, 5222.

46 C. Zhao, A. M. Bond and X. Lu, Anal. Chem., 2012, 84, 2784-2791. 47 J. Penfold and R. K. Thomas, J. Phys.: Condens. Matter, 1990, 2, 1369-1412.

48 R. J. L. Welbourn and S. M. Clarke, Curr. Opin. Colloid Interface Sci., 2019, 42, 87-98.

49 O. Arnold, J. C. Bilheux, J. M. Borreguero, A. Buts, S. I. Campbell, L. Chapon, M. Doucet, N. Draper, R. Ferraz Leal, M. A. Gigg, V. E. Lynch, A. Markvardsen, D. J. Mikkelson, R. L. Mikkelson, R. Miller, K. Palmen, P. Parker, G. Passos, T. G. Perring, P. F. Peterson, S. Ren, M. A. Reuter, A. T. Savici, J. W. Taylor, R. J. Taylor, R. Tolchenov, W. Zhou and J. Zikovsky, Nucl. Instrum. Methods Phys. Res., Sect. A, 2014, 764, 156-166.

50 M. Björck and G. Andersson, J. Appl. Crystallogr., 2007, 40, 1174-1178.

51 N. Hjalmarsson, D. Wallinder, S. Glavatskih, R. Atkin, T. Aastrup and M. W. Rutland, Nanoscale, 2015, 7, 16039-16045.

52 C. M. Johnson, E. Tyrode, S. Baldelli, M. W. Rutland and C. Leygraf, J. Phys. Chem. B, 2005, 109, 321-328.

53 Y.-L. Wang, F. U. Shah, S. Glavatskih, O. N. Antzutkin and A. Laaksonen, J. Phys. Chem. B, 2014, 118, 8711-8723.

54 N. Ji, V. Ostroverkhov, C.-Y. Chen and Y.-R. Shen, J. Am. Chem. Soc., 2007, 129, 10056-10057.

55 A. Ge, Q. Peng, L. Qiao, N. R. Yepuri, T. A. Darwish, M. Matsusaki, M. Akashi and S. Ye, Phys. Chem. Chem. Phys., 2015, 17, 18072-18078. 
56 J. Wang, Z. Paszti, M. A. Even and Z. Chen, J. Am. Chem. Soc., 2002, 124, 7016-7023.

57 G. J. Simpson and K. L. Rowlen, J. Am. Chem. Soc., 1999, 121, 2635-2636.

58 R. A. MacPhail, H. L. Strauss, R. G. Snyder and C. A. Elliger, J. Phys. Chem., 1984, 88, 334-341.

59 D. A. Beattie, S. Haydock and C. D. Bain, Vib. Spectrosc., 2000, 24, 109-123.

60 A. M. Moschovi, V. Dracopoulos and V. Nikolakis, J. Phys. Chem. B, 2014, 118, 8673-8683.

61 M. N. Garaga, M. Nayeri and A. Martinelli, J. Mol. Liq., 2015, 210, 169-177.

62 J. Joseph and E. D. Jemmis, J. Am. Chem. Soc., 2007, 129, 4620-4632.

63 J.-C. Lassegues, J. Grondin, D. Cavagnat and P. Johansson, J. Phys. Chem. A, 2009, 114, 687-688.

64 A. Wulf, K. Fumino and R. Ludwig, J. Phys. Chem. A, 2009, 114, 685-686.

65 S. Cha, M. Ao, W. Sung, B. Moon, B. Ahlström, P. Johansson, Y. Ouchi and D. Kim, Phys. Chem. Chem. Phys., 2014, 16, 9591-9601.

66 P. K. Cooper, H. Li, N. R. Yepuri, A. Nelson, G. B. Webber, A. P. Le Brun, T. A. Darwish, G. G. Warr and R. Atkin, J. Phys. Chem. C, 2018, 122, 24077-24084.

67 A. Graves, J. Electroanal. Chem. Interfacial Electrochem., 1970, 25, 349-356.

68 A. Graves and D. Inman, J. Electroanal. Chem. Interfacial Electrochem., 1970, 25, 357-372.
69 M. Z. Bazant, B. D. Storey and A. A. Kornyshev, Phys. Rev. Lett., 2011, 106, 046102.

70 T. Fujimoto and K. Awaga, Phys. Chem. Chem. Phys., 2013, 15, 8983-9006.

71 S. Maolin, Z. Fuchun, W. Guozhong, F. Haiping, W. Chunlei, C. Shimou, Z. Yi and H. Jun, J. Chem. Phys., 2008, 128, 134504.

72 K. Kirchner, T. Kirchner, V. Ivaništšev and M. V. Fedorov, Electrochim. Acta, 2013, 110, 762-771.

73 S. Jo, S.-W. Park, Y. Shim and Y. Jung, Electrochim. Acta, 2017, 247, 634-645.

74 M. Mezger, H. Schröder, H. Reichert, S. Schramm, J. S. Okasinski, S. Schöder, V. Honkimäki, M. Deutsch, B. M. Ocko, J. Ralston, M. Rohwerder, M. Stratmann and H. Dosch, Science, 2008, 322, 424-428.

75 T. L. Greaves and C. J. Drummond, Chem. Soc. Rev., 2013, 42, 1096-1120.

76 J. N. A. Canongia Lopes and A. A. H. Pádua, J. Phys. Chem. B, 2006, 110, 3330-3335.

77 Y. Wang and G. A. Voth, J. Am. Chem. Soc., 2005, 127, 12192-12193.

78 C. Tanford, The hydrophobic effect: formation of micelles and biological membranes, J. Wiley, 2nd edn, 1980.

79 A. Dolan, R. Atkin and G. G. Warr, Chem. Sci., 2015, 6, 6189-6198.

80 S. Kamalakannan, M. Prakash, M. M. Al-Mogren, G. Chambaud and M. Hochlaf, J. Phys. Chem. C, 2019, 123, 15087-15098.

81 S. Lee, Y.-S. Shon, R. Colorado, R. L. Guenard, T. R. Lee and S. S. Perry, Langmuir, 2000, 16, 2220-2224.

82 M. Salmeron, Tribol. Lett., 2001, 10, 69-79. 